\title{
ReApp - Wiederverwendbare Roboterapplikationen für flexible Roboteranlagen
}

\section{Auswirkungen der Ergebnisse aus ReApp auf betriebliche Funktionen am Beispiel eines Anwendungsfalls in der Elektroindustrie}

\author{
Ulrich Reiser, Uwe Müller, Mike Ludwig, Mathias Lüdtke \\ und Yingbing Hua
}

\subsection{ReApp in a Nutshell}

Ziel von ReApp ist es, den Entwicklungsprozess von Roboteranlagen durch die Nutzung von wiederverwendbaren Softwarebausteinen deutlich effizienter zu machen. Die Hemmnisse für wiederverwendbare Software bestehen heute vor allem in der stark heterogenen Landschaft der Robotik- und Automatisierungskomponenten, unterschiedlichen Roboterprogrammiersprachen und fehlenden Schnittstellenstandards. Um diese Hindernisse zu überwinden, sollen in ReApp nach dem Muster von Android (The Android Source Code) die Grundlagen für ein Ecosystem für die Robotik geschaffen werden. Das

\section{U. Reiser $(\triangle)$}

Fraunhofer IPA, Group Manager 326/Robot Systems, Nobelstrasse 12, D-70569 Stuttgart,

Deutschland

e-mail: ulrich.reiser@ipa.fraunhofer.de

U. Müller

InSystems Automation, Berlin, Deutschland

e-mail: mueller@insystems.de

M. Ludwig dresden elektronik ingenieurtechnik GmbH, Dresden, Deutschland e-mail: ml@dresden-elektronik.de

\section{Lüdtke}

Fraunhofer IPA, Stuttgart, Deutschland

e-mail: mathias.luedtke@ipa.fraunhofer.de

Y. Hua

Karlsruhe Institute of Technology (KIT), Karlsruhe, Deutschland

e-mail: yingbing.hua@kit.edu 
Android-Ecosystem ist aus wenigen Kernelelementen zusammengesetzt. Die Android Hardware-Abstraction Layer (HAL) definiert Schnittstellen (APIs), die von den Smartphone-Herstellern implementiert werden. Dadurch ist gewährleistet, dass alle AndroidApps auf allen Android-Smartphones lauffähig sind, obwohl in den Smartphones unterschiedliche Kameras, Displays und Sensoren verbaut sind. Die Hersteller haben den Vorteil, dass alle Apps im globalen App-Repository, dem Google App-Store, kompatibel zu ihren Smartphones sind. So lässt sich der hohe Marktanteil der Android-Systeme von über $80 \%^{1}$ erklären. Neben der HAL, die sicherlich ein Schlüsselelement des Ecosystems darstellt, sorgen Entwicklungswerkzeuge, die Android SDK Tools, für die einfache Entwicklung von neuen Apps und die Einhaltung von Standards. Für ein standardisiertes Deployment (d. h. Installation und Ausführung der Apps) sorgt die Laufzeitumgebung, Android Runtime.

Um die Zielsetzung von ReApp zu verdeutlichen, sind die einzelnen Elemente des avisierten ReApp-Ecosystems in Abb. 10.1 den einzelnen Elementen des Android-Ecosystems gegenübergestellt. Die Rolle der Android HAL wird dabei dem OpenSource Robo-

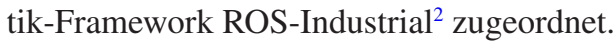

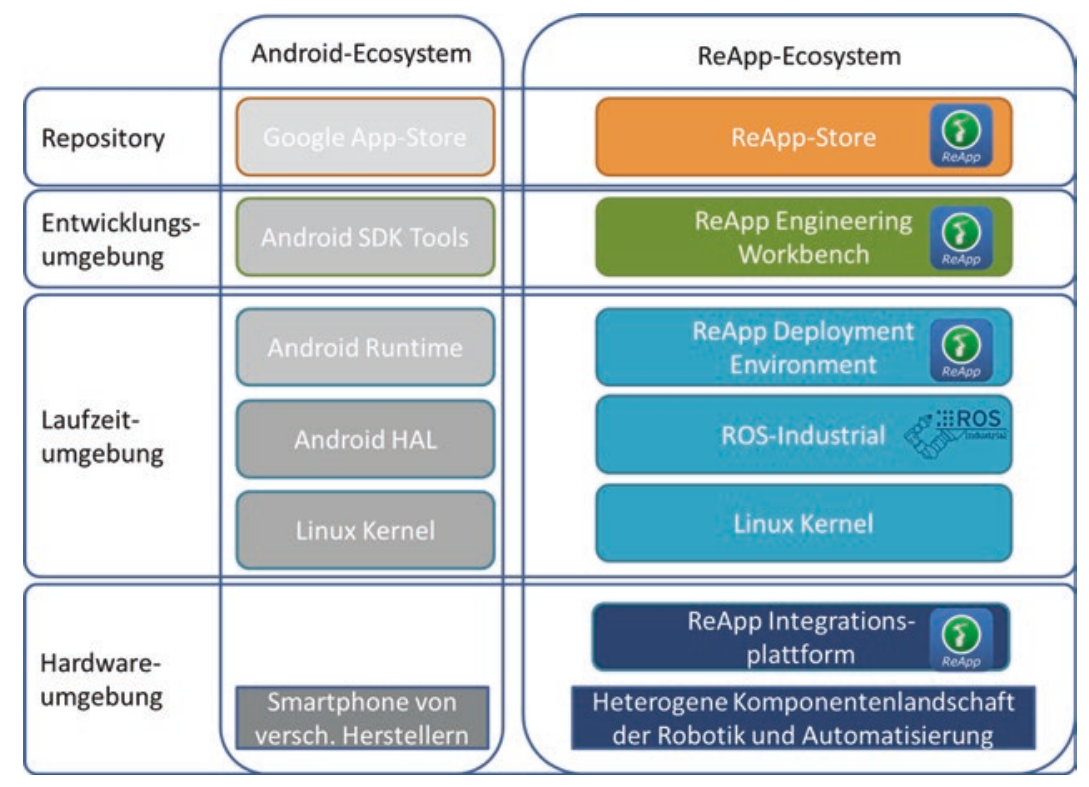

Abb. 10.1 Das ReApp-Ecosystem

\footnotetext{
${ }^{1}$ Quelle: Statista: Marktanteil von Android am Absatz von Smartphones weltweit vom 1. Quartal 2009 bis zum 2. Quartal 2015.

${ }^{2}$ www.rosindustrial.org
} 


\subsubsection{Das ReApp-Projektkonsortium}

Das ReApp-Konsortium setzt sich neben den Forschungspartnern fortiss gGmbH (FORTISS), dem FZI Forschungszentrum Informatik, dem Karlsruhe Institute of Technology (KIT) und dem Fraunhofer-Institut für Produktionstechnik und Automatisierung IPA aus einer Vielzahl von Industriepartnern zusammen. Die Technologiepartner SICK und fluid Operations AG bringen Sensorik und Cloud-Technologien in das Projekt mit ein. Die Unternehmen dresden elektronik ingenieurtechnik GmbH (DDE), BMW AG und Fischer IMF GmbH \& Co. KG werden die Ergebnisse von ReApp als Endanwender beurteilen, die Unternehmen Ruhrbotics GmbH und InSystems Automation GmbH als Systemintegratoren. Damit deckt das Konsortium alle Beteiligten ab, die für die Entwicklung von roboterbasierten Automatisierungslösungen heute eine Rolle spielen. Dies ist erforderlich, um die unterschiedlichen Aspekte und möglichen Auswirkungen des Forschungsprojekts vollumfänglich beurteilen zu können.

\subsubsection{Der ReApp-Lösungsansatz}

Um die in Abschn. 10.1.1 genannten Projektziele zu erreichen, wurden zu Beginn des Projekts die einzelnen Elemente des ReApp-Ecosystems (Abb. 10.2) konzipiert, bei der alle in der Vorhabenbeschreibung definierten Teilziele (Entwicklung von intelligenten Plug\&Produce-Komponenten, Integrationsplattform, Komposition und Konfiguration von Programmbausteinen, intuitive Entwicklungsumgebung etc.) zu einer Gesamtlösung integriert werden. Ein großer Schwerpunkt liegt dabei auf der Schaffung einer durchgängigen Entwicklungsumgebung,

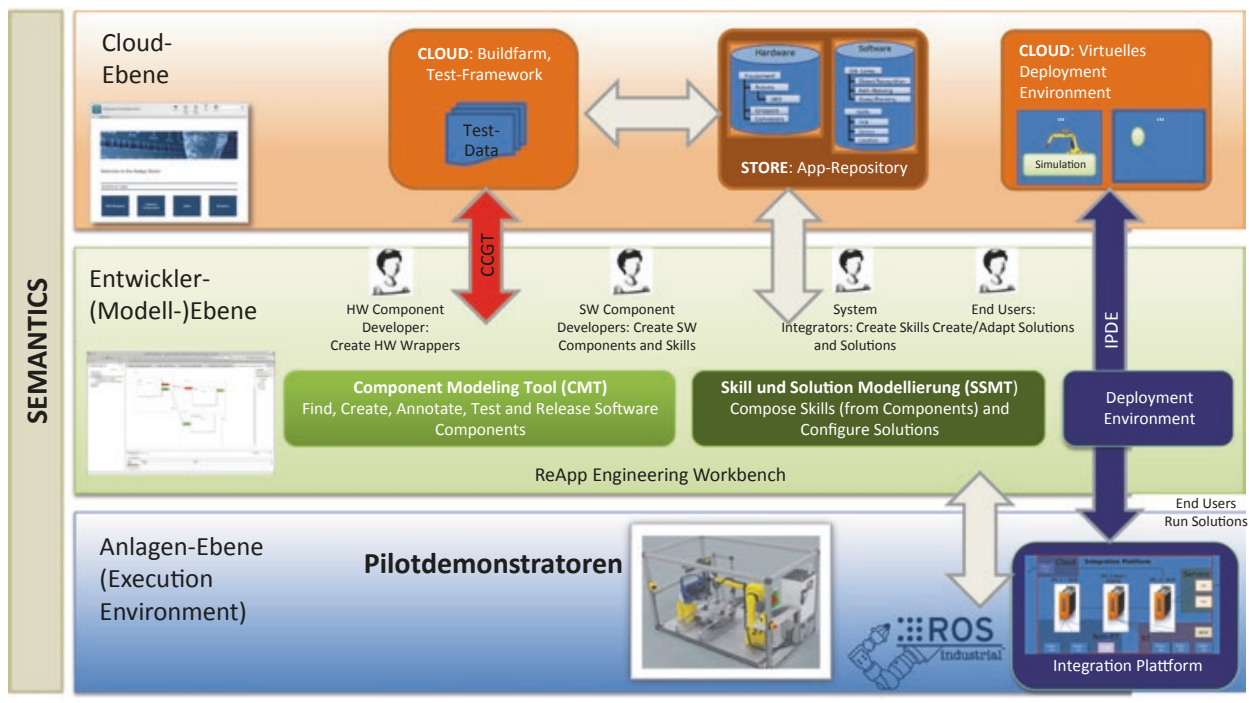

Abb. 10.2 Überblick über die einzelnen Elemente des ReApp-Ecosystems 
der ReApp Engineering Workbench, die sowohl die Verbindung zum App-Repository, dem ReApp-Store, als auch zur Ausführungsschicht und der Hardware bereitstellen muss.

Mit dem „Component Modeling Tool“ (CMT) können neue Apps, z. B. HardwareTreiber oder höherwertige Software-Komponenten, entwickelt und, nach erfolgreichem Durchlaufen einer Qualitätssicherung, dem Testframework, in den Store eingestellt werden. Das CMT stellt dabei sicher, dass die entwickelte Komponente einem standardisierten Komponentenmodell entspricht, das Eigenschaften, Strukturen und die Semantik von Komponenten definiert und dadurch die Kompatibilität der Komponenten gewährleistet. Die Komponenten werden dann automatisch bezüglich der ReApp-Ontologie (Zander et al. 2015) klassifiziert, die eine spätere Suche im Store erheblich vereinfacht.

Mit dem „Skill und Solution Modeling Tool“ (SSMT) können Apps zu komplexeren Anwendungen komponiert werden. Die Grundidee ist, dass zunächst hardwareunabhängige Fähigkeiten oder Skills erstellt werden, die eine hohe Wiederverwendbarkeit gewährleisten. Ein generischer Pick-and-Place-Skill könnte z. B. aus den folgenden Basis-Apps aufgebaut sein: einer Objektlageerkennung, die mithilfe eines Farbbildes und eines Objektmodells die Position und Lage des Objektes im Bild wiedergibt, und einer Trajektoriengenerierung, die mithilfe von Tiefendaten der Umgebung und dem Robotermodell eine kollisionsfreie Trajektorie berechnet. Soll der Skill dann für einen bestimmten Roboter und eine bestimmte Kamera wiederverwendet werden, müssen lediglich die Treiber-Apps für die Hardware hinzugefügt werden. Aus dem Skill wird so eine „Solution“. Sowohl Skills als auch Solutions können im Store bereitgestellt werden.

Schließlich kann die erstellte Solution mithilfe der ReApp-Deployment-Umgebung auf einer konkreten Anlage installiert, konfiguriert und ausgeführt werden. Dabei hilft die ebenfalls standardisierte Steuerungshardware, die sogenannte „Integrationsplattform“. Sie bietet physikalische Schnittstellen für gängige Feldbusse, sodass Robotersteuerungen oder SPS-Steuerungen einfach angebunden werden können. Damit ist eine durchgängige Deployment-Kette vom Store bis auf die Steuerung geschaffen, die vom Entwickler abstrahiert wird. Das bedeutet, dass der Systemintegrator weder die üblichen Schnittstellenanpassungen noch Modifikationen für die Steuerungsarchitektur durchführen muss. Er kann sich darauf verlassen, dass alle Apps im Store, die über die ReApp-DeploymentUmgebung auf die Integrationsplattform installiert wurden, auch kompatibel und lauffähig sind. Wie in Abb. 10.2 dargestellt, kann das Deployment auch zunächst in einer virtuellen Laufzeitumgebung in der Cloud getestet werden.

Die ReApp Engineering Workbench unterstützt somit den gesamten Lebenszyklus von roboterbasierten Automatisierungsanlagen: Konzeption, Simulation und Test, Aufbau und Inbetriebnahme, Konfiguration und Rekonfiguration, Deployment und Operation.

\subsubsection{Auswirkungen auf betriebliche Funktionen}

Da Industrieroboterzellen heute in aller Regel durch Systemintegratoren konzipiert, aufgebaut, programmiert, eingerichtet, in Betrieb genommen und gewartet werden, ist diese Berufsgruppe sicherlich am unmittelbarsten und am umfangreichsten von den 
ReApp-Lösungskonzeptionen betroffen. Durch ReApp soll eine Plattformtechnologie geschaffen werden, mit der eine um ein Vielfaches erhöhte Wiederverwendbarkeit bereits existierender Lösungen erzielt werden soll. Der Entwicklungsprozess wird sich daher vor allem in der Designphase stark ändern: Anstatt Hardwarekomponenten für den Aufbau der Automatisierungszelle nur nach Erfahrung und Wunsch des Kunden auszusuchen, wird in Zukunft die technische Eignung für die Anwendung des Kunden eine größere Rolle als bisher spielen, da der Entwicklungsaufwand durch die Plattformtechnologie weitgehend unabhängig von der Wahl des Herstellers ausfällt.

Während große Endanwender wie BMW eher indirekt durch den geänderten Entwicklungsprozess bei den Systemintegratoren tangiert werden, könnten kleine und mittelständische Unternehmen in die Lage versetzt werden, auch für kleine Stückzahlen roboterbasierte Automatisierung einzusetzen.

Durch die ReApp-Infrastruktur sollen die folgenden betrieblichen Rollen durch standardisierte Schnittstellen und verbesserte Datendurchgängigkeit besser separiert werden:

- Entwickler von Automatisierungskomponenten (z. B. Sensoren, Aktoren, Steuerungen)

- Entwickler von Softwarekomponenten (z. B. Bilderkennung, HMI etc.)

- Entwickler von Simulationssoftware/Simulationsmodellen

- Anlagenentwickler/Systemintegratoren

- Entwickler von Applikationssoftware

Neben Entwicklerfunktionen wird ReApp auch Auswirkungen auf Endanwender haben. Zum einen soll durch die verbesserte Effizienz in der Anlagenentwicklung und -einrichtung eine Erhöhung des Automatisierungsgrads auch bei kleinen Stückzahlen ermöglicht werden. Monotone (und damit fehleranfällige) sowie einfache Tätigkeiten werden so reduziert. Zum anderen soll Endanwendern durch entsprechende Schnittstellen eine größere Möglichkeit gegeben werden, Systemparameter zu konfigurieren und die Anlage in gewissem Maße eigenständig umzurüsten.

In ReApp wurden im ersten Projektjahr drei Pilotdemonstratoren spezifiziert, die unterschiedliche Aufgaben der Endanwender behandeln. Dazu gehören das Kommissionieren bei einem Automobilzulieferer, das Löten für einen Elektronikhersteller und die Türfertigung für einen Autohersteller. In Pilotdemonstratoren ändern sich die Arbeitsabläufe für die folgenden Funktionen/Tätigkeiten:

- Maschinenbediener

- Bandarbeiter in der Automobilproduktion

- Monteure

- Kommissionierer

- Elektronikfachkräfte/Lötfachkräfte

Im Folgenden wird anhand eines der drei Pilotdemonstratoren erörtert, welche Auswirkungen sich durch den Einsatz einer roboterbasierten Automatisierungslösung ergeben und welche Rolle die Projektergebnisse von ReApp dabei spielen. 


\subsection{Anwendungsfall: Bestücken in der Elektroindustrie - "Automatisches Verlöten von LED-Stripes"}

\subsubsection{Unternehmen}

Die dresden elektronik ingenieurtechnik GmbH begann 1990 mit drei Mitarbeitern und entwickelte sich während der letzten 25 Jahre kontinuierlich zu einem Komplettanbieter für Elektronikentwicklung und -fertigung mit über 80 Arbeitsplätzen. Während am Anfang vorwiegend Geräte für die Industrieautomatisierung hergestellt wurden, hat sich das Portfolio von dresden elektronik mittlerweile auf viele Businessbereiche ausgedehnt, u. a. Entwurf und Entwicklung von Hard- und Software, Module für die drahtlose Kommunikation, Lichtsignalanlagen für die Verkehrstechnik und Bestückung von Leiterplatten in SMD und Durchstecktechnologie.

Die steigende Komplexität der Geräte und das immer breiter werdende Spektrum an Geräteoptionen führt dazu, dass die Anzahl der bestückten Leiterplatten von einem Typ immer weiter sinkt, obwohl die Gesamtbestellmenge gleich bleibt oder gar steigt.

Die Bestückung von Leiterplatten - speziell von SMD-Prozessen - ist schon hoch automatisiert. Allerdings ist die Bestückung von Durchsteckbauelementen auf Leiterplatten immer noch zum großen Teil Handarbeit. Da fast jede Leiterplatte Durchsteckelemente aufweist, erfordert diese Bestückung - die variantenspezifisch meist nach der größtenteils automatisierten SMD-Bestückung erfolgt - eine manuelle Bearbeitung. Obwohl durch Schwalllöten und Selektivlöten schon automatisierte Lötprozesse für Durchsteckbauelemente genutzt werden, ist in vielen Fällen zusätzlich auch ein Handverlöten einiger Bauelemente erforderlich. Damit wird Durchsteckbestückung zu einem entscheidender Kostenfaktor in der Gesamtkalkulation, in der Handlöten die teuerste Art der Bestückung darstellt.

Die Entwicklung, Baugruppen in mehreren Varianten herzustellen, führt dazu, dass die Losgrößen immer weiter sinken und damit die Produktion eine immer flexiblere Ausstattung benötigt, welche schnell und effektiv an das nächste Produkt oder die nächste Variante angepasst und rekonfiguriert werden kann. Die benötigte Einrichtzeit aller Maschinen ist mittlerweile zu einem der wichtigsten Kostenfaktoren für eine konkurrenzfähige Leiterplattenbestückung im Kleinserienbereich geworden.

Um die notwendigen kostenintensiven manuellen Prozesse auch am Standort Deutschland konkurrenzfähig anbieten zu können, braucht es neue Automatisierungslösungen mit dem Fokus auf kurze Einrichtzeiten und hohe Flexibilität.

Im Betrieb sind neben Entwicklungsingenieuren vor allem Elektroniker, Mechatroniker und Elektronikfacharbeiter in der Produktion beschäftigt.

\subsubsection{Beschreibung des Anwendungsfalls}

Das für das Pilotprojekt gewählte Produkt ist Teil von handelsüblichen LED-Leuchten. Diese Leuchten werden in unterschiedlichen Längen, Leuchtfarben und Verbrauchsklassen angeboten. Außerdem gibt es noch Optionen für kundenspezifische Anschlusslängen und Endstück-Ausführungen. 


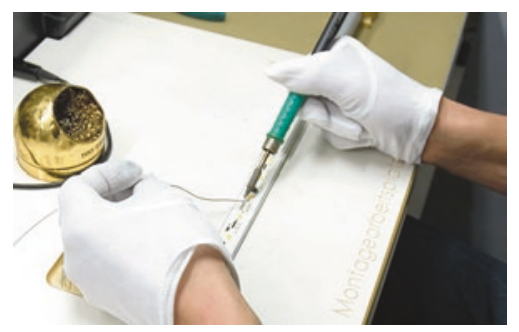

Abb. 10.3 Manueller Lötprozess von LED-Leuchtstreifen

Diese breite Palette von LED-Leuchten basiert auf Leiterplatten, die unterschiedlich bestuickt und miteinander verbunden werden. Die Leiterplatte wird in die Trageschiene mit auftragsspezifischer Länge eingefädelt, welche in einer Montage- und Lötlehre fixiert wurde. Nach dem Einschub einer weiteren Leiterplatte in die Lötlehre werden die Leiterplatten so ausgerichtet, dass sie aneinanderstoßen. Beide Leiterplatten müssen nun durch zwei kurze Drähte an zwei Stellen durch Löten von Hand miteinander verbunden werden (s. Abb. 10.3). Dabei muss sowohl die mechanische Ausrichtung als auch der Abstand der Leiterplatten zueinander exakt ausgerichtet werden. Nach dem Verlöten werden die beiden verbundenen Leiterplatten weiter in die Tragschiene eingeschoben und die nächste Leiterplatte in die Montage- und Lötlehre eingeschoben. Dieser Prozess wiederholt sich so lange, bis die für diese LED-Leuchte erforderliche Anzahl an Leiterplatten miteinander verbunden ist.

Danach erfolgt die manuelle Montage des Endstückes, um die Leiterplatten in der Tragschiene zu fixieren. Zum Abschluss des Bestückungsprozesses wird das Anschlusskabel entsprechend der Bestelloption per Hand angelötet. Die Anschlusskabel selbst werden in einem separaten Schritt konfektioniert.

Das Verlöten von LED-Stripes (LED-Leisten) wird zurzeit an einem Arbeitstisch auftragsbezogen durch einen Mitarbeiter durchgeführt (s. Abb. 10.4).

\subsubsection{ReApp-Lösungsansatz: mobile, roboterbasierte Lötstation}

Der Fokus bei der Entwicklung einer Automatisierungslösung lag neben wirtschaftlichen Aspekten hauptsächlich darauf, die manuelle Arbeit zu erleichtern und die Qualität zu

Abb. 10.4 Bisheriger Arbeitsplatz mit manueller Löt-Station und Hilfswerkzeugen (Quelle DDE)

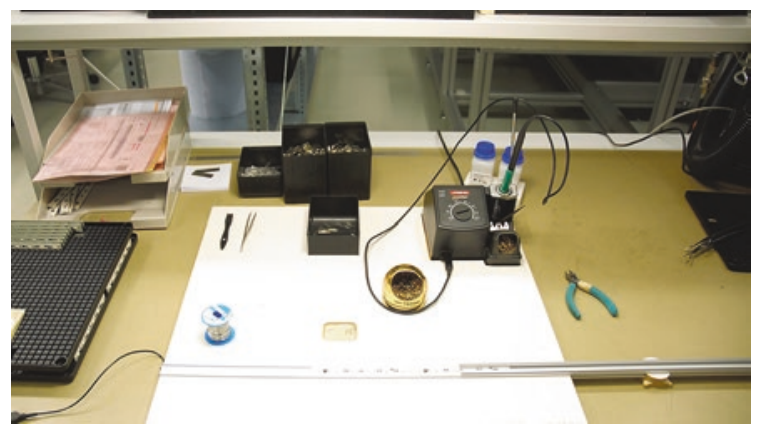


erhöhen. Bisher musste durch den Mitarbeiter bei der Herstellung von Lötverbindungen sowohl der Lötkolben als auch der Lötdraht zugeführt werden. Gleichzeitig wurde auch der Draht oder das Kabelende auf dem Lötpad platziert und fixiert - meist durch Niederhalten mit der heißen Spitze des Lötkolbens. Beim Platzieren des Drahtes oder der Kabelenden können so sehr schnell ,kalte“ Lötstellen entstehen.

Da das manuelle Löten typischerweise in kleinen Losgrößen erfolgt, musste auch eine sehr flexible Lösung angedacht werden, um sehr schnell unterschiedliche Lötaufgaben teilweise an verschiedenen Arbeitsplätzen - durchführen zu können. Hier ist besonders ein möglichst geringer Rüstaufwand gefordert, um die Herstellkosten für die Produktion in kleinen Losgrößen wirtschaftlich gestalten zu können.

Der Lösungsansatz war hier, eine mobile Einheit zu schaffen, die mit einem Leichtbauroboter, allen nötigen Steuereinheiten und auch Sicherheitseinrichtungen ausgestattet werden soll (s. Abb. 10.5). Ein automatischer Lötkolben mit Lötdrahtvorschub als Prozesswerkzeug vereinigt die hohe Präzision eines Roboters mit der Möglichkeit der Herstellung von reproduzierbaren Lötverbindungen.

Beim automatischen Kolbenlöten wird der Kolben mit seinem pneumatischen Hub durch den Roboter auf die Lötstelle aufgesetzt. Wenn die Lötstelle auf Löttemperatur erwärmt ist, wird mit dem automatischen Lötdrahtvorschub der Lötdraht zugeführt. Anschließend wir der Lötdrahtvorschub wieder zurückgezogen. Die Lötspitze verbleibt noch kurze Zeit auf der Lötstelle, damit das Lot richtig verläuft und sich ein Meniskus an der Lötstelle ausbildet.

Durch den Einsatz eines Leichtbauroboters UR 10 mit nur geringen Bahngeschwindigkeiten und auch nur kurzen Bewegungsabläufen konnten die Sicherheitseinrichtungen zum Schutz des Anwenders und anderer Personen überschaubar gestaltet werden. Neben ausklappbaren Seitenflügeln, die Eingriffe in den Prozessraum verhindern, wurde die Löteinheit gegen unabsichtliches Berühren durch eine Einhausung geschützt. Da der Lötkolben allerdings auch nach dem Lötprozess für einige Zeit heiß bleibt und damit eine potenzielle Gefahrenquelle darstellt, wurde hier ein Mechanismus entwickelt, welcher die Lötspitze in die Einhausung zurückzieht. Dies erfolgt auch bei Auslösung der Not-Aus-Funktion.

Abb. 10.5 Mobile Roboter-Einheit mit UR 10 und Löteinheit (Quelle LP Montagetechnik)

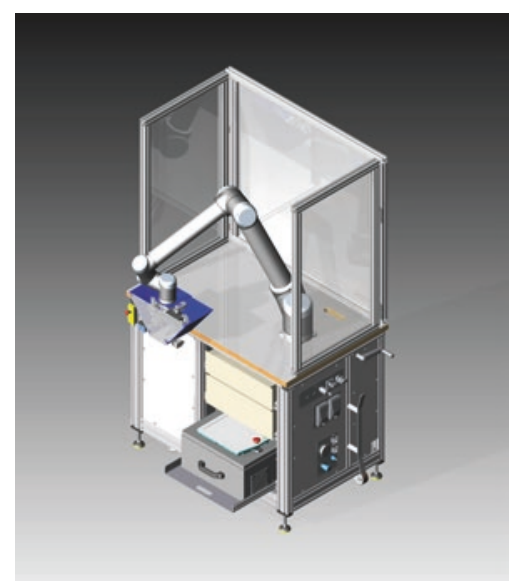


Ein Abstandssensor erkennt den Abstand zur Lötstelle, Miniaturschaltleisten vermindern zudem die Gefahr des Quetschens für den Bediener. Die Ausrichtung der LED-Stripes wird von einer 2D-Kamera überwacht. Auch eine falsche Ausrichtung der LED-Stripes sowie die Positionierung zueinander werden so erkannt und Fehler vermieden.

Die Software-Architektur für die beschriebene Funktionalität ist in Abb. 10.6 abgebildet. Die grünen Achtecke repräsentieren einzelne Skills, die wiederum aus weiteren grundlegenderen Apps zusammengesetzt sind. So besteht z. B. der Skill „Soldering Localization“, der die Lage der LED-Stripes ermittelt, aus einer Kameratreiber-App und einer Objekterkennungs-App. Die Objekterkennungs-App erhält dabei Bildmuster für die LED-Stripes als Parameter und gibt die Pose des erkannten Stripes zurück. Im „Soldering Controller" erfolgt die Koordination der einzelnen Skills, d. h. die Ablaufsteuerung des gesamten mobilen Lötroboters.

Die mobile Robotereinheit kann an jeden Arbeitsplatz angedockt werden. Nach Herstellen der Energieversorgung und nur kurzem Einlernen der neuen Aufgabe kann die Station zur Unterstützung für manuelle Lötaufgaben eingesetzt werden. Das Anlernen der neuen Aufgabe erfolgt grundsätzlich über die ReApp-Entwicklungsumgebung und die in ReApp modellierten Apps.

\subsection{Auswirkungen auf die betrieblichen Abläufe und die Personalstruktur}

\subsubsection{Auswirkungen für den Endanwender}

Auf die Personalstruktur ergeben sich unmittelbare Auswirkungen durch den Einsatz des Roboters: Die erforderliche Qualifikation des für den Fertigungsprozess einzusetzenden Personals kann durch die automatisierten Lötplätze reduziert werden. Wo vorher HandlötFacharbeiter eingesetzt werden mussten, können nun niedriger qualifizierte Mitarbeiter als Bediener eingesetzt werden. Da der Bediener vorbereitende Tätigkeiten neben dem nun automatisierten Lötvorgang zeitneutral abarbeiten kann, werden die Montagezeit und dadurch die Stückkosten reduziert. Das Konzept der Zusammenarbeit zwischen Werker und Roboter ist in Abb. 10.7 dargestellt.

Im beschriebenen Anwendungsfall werden sich somit zwar die Produktionsabläufe durch den Einsatz der mobilen Roboter-Lötstation nicht per se grundlegend ändern, durch die somit tendenziell homogenere Personalstruktur können Produktionsschwankungen jedoch flexibler als früher ausgeglichen werden. Diese zusätzliche Flexibilität in der Planung erhöht die Termintreue bei der Abarbeitung der Aufträge im Unternehmen.

Zwar eignen sich nicht alle Leiterplatten zur Übergabe an den Roboter, da neben der Umprogrammierung des Roboters auch noch weitere produktspezifische Vorbereitungen getroffen werden müssen, wie z. B. die Anfertigung einer Halterung für die Leiterplatte, damit diese für den Roboter lötbar gelagert ist. Sofern sich der mobile Lötroboter jedoch in weniger als 30 Minuten und ohne spezielles Fachwissen umprogrammieren lässt, wird 

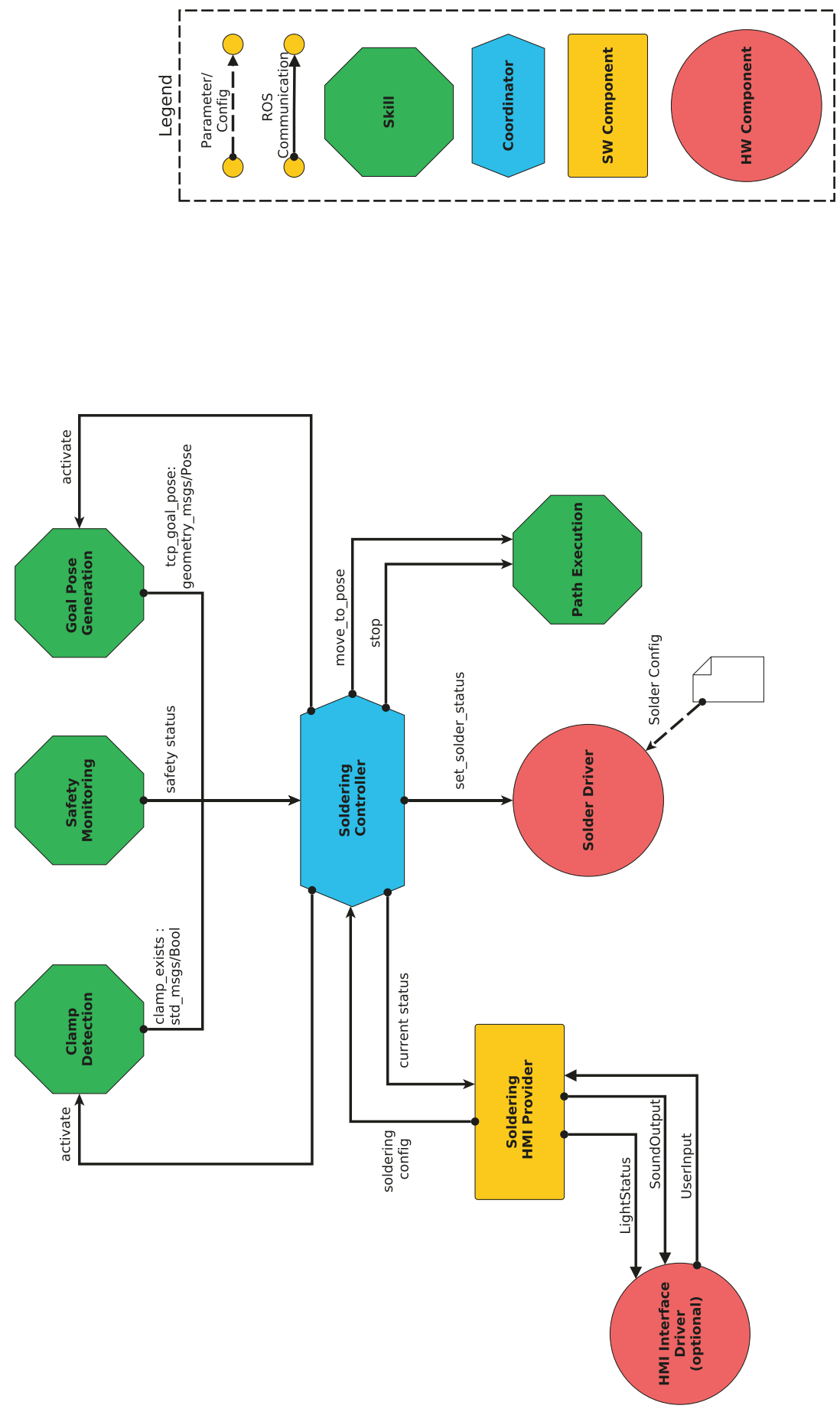

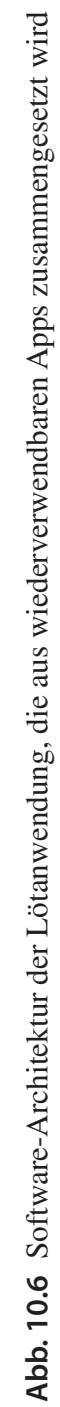


Abb. 10.7 Arbeitsplatz mit mobiler roboterbasierter Löteinheit

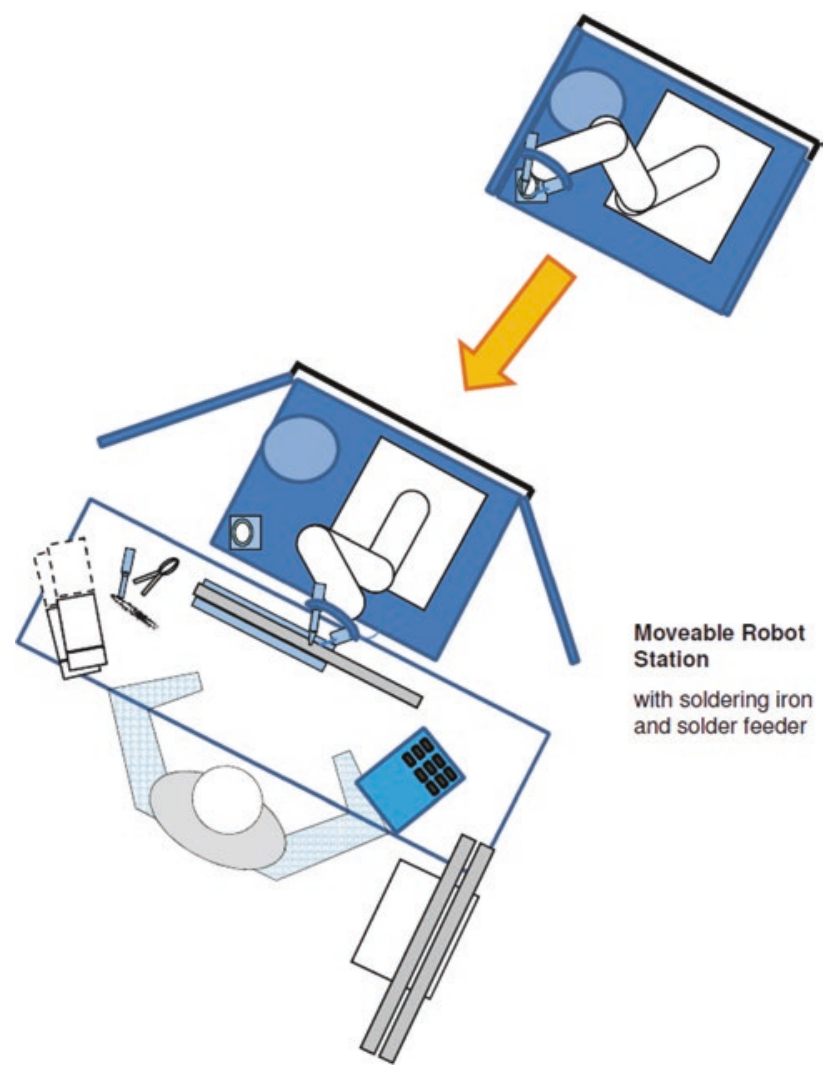

eine wirtschaftliche Einsetzbarkeit bereits für Stückzahlen ab ca. 100 Stück erwartet. Produkte, welche sich bisher durch die manuelle Herstellung nicht gerechnet haben, könnten weiter oder wieder in Deutschland gefertigt werden. Grundsätzlich gilt: Je geringer der Einrichtungsaufwand des Roboter auf das aktuelle Produkt ist (inkl. der dazu notwendigen Einrichtungsschritte wie Kalibrierungssequenz starten, Artikelnummer wählen etc.), desto flexibler und für desto geringere Stückzahlen lässt sich die Lösung einsetzen. Wo bisher aus Kostengründen keine spezielle Automatisierungslösung infrage kam, kann nun der roboterunterstützte Lötarbeitsplatz helfen, eine ähnliche Kostenreduzierung wie eine spezielle Automatisierungslösung zu erreichen. Gleichzeitig ist ein Roboter wesentlich flexibler und kann daher von mehreren Personen genutzt werden.

Eine weitere Auswirkung ergibt sich durch die gleichbleibend gute Qualität des automatisierten Lötprozesses. Selbst bei gut ausgebildeten Fachkräften schwankt die Lötqualität, sodass beim Roboterlöten deutlich weniger Nacharbeiten als bisher zu erwarten sind. In der Qualitätssicherung können somit perspektivisch Kosten gespart werden.

Die nachfolgende Tabelle (Tab. 10.1) gibt die Einschätzung der Auswirkungen eines Einsatzes der roboterbasierten Lötstation auf die betrieblichen Funktionen wieder. 
Tab. 10.1 Auswirkungen auf die betrieblichen Funktionen

\begin{tabular}{l|l|l|l}
\hline & $\begin{array}{l}\text { Gering qualifizierte } \\
\text { Mitarbeiter }\end{array}$ & Fachkräfte & Gruppenleiter/Ingenieur \\
\hline Bedarf & ++ & - & - \\
\hline Problemlösung & - & - & - \\
\hline Monotone Aufgaben & -- & -- & - \\
\hline Komplexe Aufgaben & - & + & + \\
\hline Planen & 0 & 0 & + \\
\hline Kontrolle & - & - & -- \\
\hline Lernen, informell & 0 & + & + \\
\hline Lernen, formell & + & + & + \\
\hline Selbstbestimmung & - & + & 0 \\
\hline Optimierung & - & + & + \\
\hline Kooperation & + & + & + \\
\hline Kommunikation & + & + & + \\
\hline Interdisziplinarität & 0 & + & + \\
\hline IT-Kenntnisse & + & 0 & + \\
\hline
\end{tabular}

Legende: - - wird viel weniger, - wird weniger, 0 bleibt gleich, + wird mehr, ++ wird viel mehr

Zusammengefasst lässt sich sagen: Eine Änderung der Personalstruktur wird bereits bei einem, aber insbesondere beim Einsatz mehrerer roboterunterstützter Arbeitsplätze erwartet, da diese dann von geringer ausgebildetem Personal bedient werden können. Gleichzeitig steigen die Anforderungen an die Arbeitsvorbereitung, die durch den Gruppenleiter oder Ingenieur einmalig bei der Einführung des Produkts in die Fertigung durchgeführt wird, da hier das Einrichten der roboterbasierten Lötstation mit berücksichtigt werden muss.

\subsubsection{Auswirkungen für den Systemintegrator}

Im oben genannten Beispiel können Änderungen und Anpassungen an der mobilen Roboterstation durch Hinzufügen oder Löschen von Skills oder der Modifikation des Ablaufs im Koordinator vorgenommen werden. Eine grundlegende Neuentwicklung ist nicht erforderlich, selbst wenn mehrere Skills (z. B. automatisierte Erkennung des Lötdrahts) hinzugefügt werden sollen. Der Schwerpunkt wird somit von der Umsetzung in Richtung Konzeption verlagert.

Für die Systemintegratoren bedeutet der mögliche Einsatz der ReApp-Infrastruktur eine Verringerung des Installations- und Programmieraufwandes bei Automatisierungsprojekten 
und dadurch die Möglichkeit von attraktiveren, wirtschaftlicheren Lösungskonzepten gerade für mittelständische Endanwender wie dresden elektronik.

Die Bedingung dafür ist ein Wandel der Qualifikation der Mitarbeiter von Systemintegratoren. Es werden weniger Softwareexperten mit fundierten Programmiererfahrungen und Hochsprachenkenntnis benötigt, vielmehr werden Erfahrungen gerade im Bereich der Konzeptionierung von Mensch-Maschine-Kooperation, Anwendungsingenieure mit Kenntnissen in visueller Technologie (z. B. Bilderkennung) oder Erfahrungen in der Navigation autonomer mobiler Systeme benötigt.

\subsection{Zusammenfassung und Fazit}

Mit ReApp werden roboterbasierte Automatisierungslösungen immer mehr auch für kleine Losgrößen und somit für kleine und mittelständische Unternehmen einsetzbar. Die Programmierung und Einrichtung wird deutlich vereinfacht und kann in Zukunft zunehmend vom Endanwender direkt durchgeführt werden.

Diese Automatisierungssysteme werden Auswirkungen auf die Personalstruktur haben. Vor allem repetitive Prozesse, die eine hohe Genauigkeit oder Zuverlässigkeit erfordern, werden verstärkt durch Roboter durchgeführt werden. Dadurch wird in der Regel die Anzahl von ungelernten Mitarbeiten zugunsten von Personal mit Qualifizierung im Bereich Automatisierungstechnik (z. B. Roboterprogrammierern) tendenziell zurückgehen. Wie das Anwendungsbeispiel „Automatisiertes Löten“ in diesem Kapitel zeigt, kann jedoch auch der umgekehrte Fall auftreten. Durch den Einsatz einer roboterbasierten Lötstation können hochqualifizierte Fachkräfte durch gering qualifizierte „Roboterbediener“ ersetzt werden. Voraussetzung ist in diesem Beispiel jedoch die einfache Programmierbarkeit der roboterbasierten Lötstation. Sind spezielle Kenntnisse zur Umprogrammierung erforderlich oder dauert der Einrichtprozess insgesamt zu lange, ist ein wirtschaftlicher Einsatz des Roboters gefährdet.

Roboter werden in Zukunft nicht mehr in herstellerspezifischen Roboterprogrammiersprachen programmiert, sondern mithilfe von Softwarewerkzeugen, die wiederverwendbare Programmbausteine kombinieren und konfigurieren. Systemintegratoren werden sich deshalb noch stärker von reinen Roboterprogrammierern zu Systemexperten mit Kenntnissen u. a. in Mensch-Roboter-Kooperation, Bilderkennung und weiteren höherwertigen Funktionalitäten wandeln.

\subsection{Danksagung}

Dieser Beitrag ist im Rahmen des Projekts „Wiederverwendbare Roboterapplikationen für flexible Roboteranlagen basierend auf Industrial-ROS“ entstanden. Das Projekt wird vom Bundesministerium für Wirtschaft und Energie (BMWi) unter dem Kennzeichen 01MA13001 gefördert und vom DLR-Projektträger betreut. 


\section{Literatur}

The Android Source Code. https://source.android.com/source/index.html. Zugegriffen: 21. Dez. 2015. Zander, S., Heppner, G., Neugschwandtner, G., Awad, R., Essinger, M., \& Ahmed, N. (2015). „A Model-Driven Engineering Approach for ROS using Ontological Semantics", 6th International Workshop on Domain-Specific Languages and models for ROBotic systems (DSLRob-15) co-located with 2015 IEEE/RSJ International Conference on Intelligent Robots and Systems (IROS), Hamburg, Germany.

Open Access Dieses Kapitel wird unter der Creative Commons Namensnennung 4.0 International Lizenz (http://creativecommons.org/licenses/by/4.0/deed.de) veröffentlicht, welche die Nutzung, Vervielfältigung, Bearbeitung, Verbreitung und Wiedergabe in jeglichem Medium und Format erlaubt, sofern Sie den/die ursprünglichen Autor(en) und die Quelle ordnungsge-mäß nennen, einen Link zur Creative Commons Lizenz beifügen und angeben, ob Änderungen vorgenommen wurden.

Die in diesem Kapitel enthaltenen Bilder und sonstiges Drittmaterial unterliegen ebenfalls der genannten Creative Commons Lizenz, sofern sich aus der Abbildungslegende nichts anderes ergibt. Sofern das betreffende Material nicht unter der genannten Creative Commons Lizenz steht und die betreffende Handlung nicht nach gesetzlichen Vorschriften erlaubt ist, ist für die oben aufgeführten Weiterverwendungen des Materials die Einwilligung des jeweiligen Recht-einhabers einzuholen.

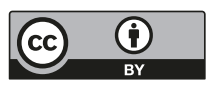

